\title{
Synthesis of Mesoporous Silica Materials from Incineration Bottom Ash for the Removal of Toluene
}

\author{
Zhen-Shu Liu and Hai-Ming Lan
}

\begin{abstract}
In Taiwan, most of the municipal solid waste is incinerated, and more than 930 thousand tons of bottom ash is produced in 2014. Incineration bottom ash contains major element of silicon that could be as a silicon source for the synthesis of mesoporous materials. Our previous study investigated the conditions for alkaline fusion to extract silica from incinerator bottom ash and verified that the high specific surface area of mesoporous silica could been synthesized from bottom ash. To expand the application of mesoporous silica materials synthesized from bottom ash, the effects of operation conditions of adsorbent weight $(0.2,0.5,0.8,1.5 \mathrm{~g})$, adsorption temperature $\left(25,30,35^{\circ} \mathrm{C}\right)$ and toluene concentrations $(900$, $1100,1300,1500 \mathrm{ppm}$ ) on toluene removal were determined in this study. Moreover, the gas flow and the oxygen content were 500 scem and $10 \%$, respectively. The mesoporous materials synthesized from bottom ash possessed the best adsorption capacity of toluene (ca. $124 \mathrm{mg} \mathrm{g-1}$ ) at the toluene concentration of $1100 \mathrm{ppm}$ and at the reaction temperature of $25^{\circ} \mathrm{C}$. The results showed that the mesoporous silica prepared with incinerator bottom ash have potential to be used as an adsorbent for the removal of toluene.
\end{abstract}

Index Terms-Adsorption, incineration bottom ash, mesoporous materials, toluene.

\section{INTRODUCTION}

Mesoporous silica materials exhibited high specific surface area, and good thermal and hydrothermal stabilities have been used extensively as catalysts and adsorbents [1]-[4]. Silica is the main raw material to synthesize these mesoporous silica materials. However, silica derived from inorganic silicates (such as sodium silicate) is expensive. Therefore, an alternative source of silica must be found. In $1985, \mathrm{SiO}_{2}$ and $\mathrm{Al}_{2} \mathrm{O}_{3}$ were firstly extracted from coal fly ash using an alkaline hydrothermal reaction [5]. Then the $\mathrm{SiO}_{2}$ and $\mathrm{Al}_{2} \mathrm{O}_{3}$ were used to synthesize microporous molecular sieves. Subsequently, many studies investigated the synthesis of mesoporous silica materials using silica source from coal fly ash [6]-[13]. Lee et al. indicated that the fly ash with high silica content has the potential for the synthesis of nanoporous materials, such as zeolites, mesoporous silica materials [8]. However, processing of mesoporous silica materials from incinerator bottom ash is obscure. The ash composition, pretreatment procedures, and hydrothermal synthesis conditions would affect the purity and yield rate of

Manuscript received September 12, 2018; revised December 7, 2018.

Zhen-Shu Liu and Hai-Ming Lan are with Department of Safety, Health and Environmental Engineering, Ming Chi University of Technology, New Taipei City, Taiwan (e-mail: zsliu@mail.mcut.edu.tw).

Zhen-Shu Liu is also with Chronic Diseases and Health Promotion Research Center, Chang Gung University of Science and Technology, Puzi, Chiayi 61363, Taiwan. the synthesized nanoporous materials.

Incinerator bottom ash containing a large amount of silica can be used as a raw material for synthesizing mesoporous silica materials [14], [15]. This would not only increase the recycling rate of bottom ash but also reduce the cost of mesoporous silica materials. In Taiwan, the recovery rate of incineration bottom ash is approximately $77 \%$ in 2014 , and $23 \%$ of bottom ash was disposed in landfill. Seeking new recycling techniques to increase the recovery rate and value added of bottom ash is important. Therefore, mesoporous silica materials synthesized from incinerator bottom ash was investigated in our previous study [14].

Our previous study verified that the high specific surface area of $992 \mathrm{~m}^{2} / \mathrm{g}$ of mesoporous silica could been synthesized from incineration bottom ash. The mesoporous silica also has been used for the removal of heavy metals from waste water [14]. It has potential for the synthesis of mesoporous silica from bottom ash and the application of mesoporous silica in the removal of pollutants. Therefore, the synthesis of mesoporous silica materials from incineration ash for the removal of toluene in a fluidized-bed reactor is investigated in this study.

\section{METHOD}

The bottom ash used in this study was obtained from an incinerator in New Taipie City, Taiwan. The bottom ash was completely dried at $105^{\circ} \mathrm{C}$ in an oven and then crushed, ground, and sieved $(<0.35 \mathrm{~mm})$. The ash less than $0.35 \mathrm{~mm}$ in size was mixed with $\mathrm{Na}_{2} \mathrm{CO}_{3}$ in a certain ratio, and then placed it in a high-temperature furnace at $900^{\circ} \mathrm{C}$ for $15 \mathrm{~min}$ for alkali fusion. The fused mass was dissolved in deionized water and placed in an oven at $105^{\circ} \mathrm{C}$ for $24 \mathrm{~h}$ for hydrolysis reaction. Subsequently, the mixed solution was filtered to obtain the supernatant. The templating agent for synthesizing mesoporous materials in this study was stirred and mixed with deionized water at $45^{\circ} \mathrm{C}$ using cetyltrimethyl ammonium bromide (CTAB). The aqueous CTAB solution was slowly introduced into the silica-containing supernatant solution. Then, $1 \mathrm{M}$ sulfuric acid was added into the mixture until the $\mathrm{pH}$ value was 10 . Subsequently, the solution was poured into an PP bottle, which was placed in an oven at $105^{\circ} \mathrm{C}$ for hydrothermal reaction, and after being cooled to room temperature and filtrated. The filtered solid was washed with deionized water and placed in an oven for drying, and then placed in a high-temperature furnace for calcination. After cooling, the filtered solid yielded mesoporous silica.

The physical and chemical characteristics of mesoporous materials were analyzed using various instruments. To study the effect of the porosity of the mesoporous silica materials 
synthesized from bottom ash on toluene adsorption, an ASAP2020 vacuum volumetric sorption instrument was used to determine the porosities and BET surface area of the synthesized mesoporous silica materials. The specific surface area was determined by BET method. Since the micropore volumes of the silica materials was zero, the mesopore volumes (pore size: $2-50 \mathrm{~nm}$ ) were the total pore volumes at a relative pressure of 0.98 . A transmission electron microscope (TEM) (JEM-2100) and a field-emission scanning electron microscopy (FE-SEM) (JEOL JSM-7600 F) were used to analyze the pore structure and morphologies of the mesoporous silica materials, respectively. An X-ray powder diffraction (XRD) system (SIEMENS D5000) was also used to observe whether the mesoporous silica has the hexagonal pore structure. The stability of the mesoporous silica can be determined by a thermogravimetric Analyzer (TGA) (Perkin Elmer STA6000).

TABLE I. EFFECTS OF VARIOUS ADSORPTION CONDITIONS ON THE ADSORPTION CAPACITY OF TOLUENE

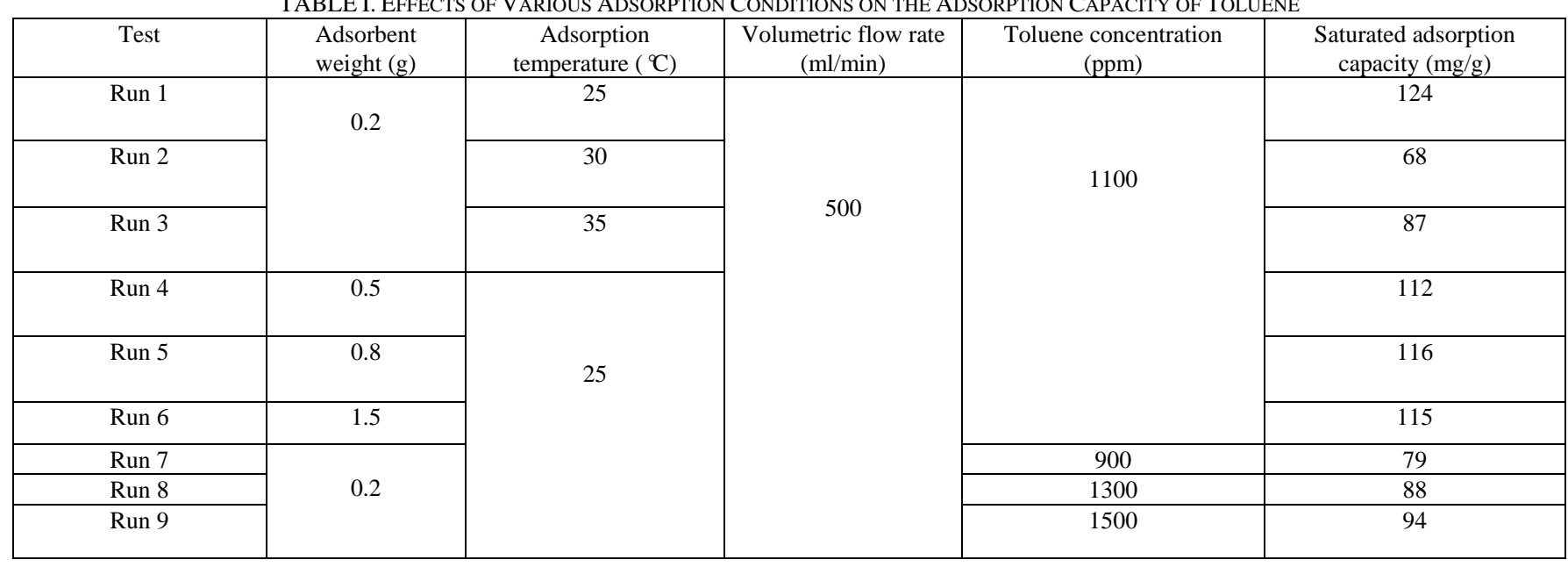

Toluene adsorbed onto mesoporous silica was carried out in a fixed-bed reactor. Fig. 1 shows the experimental setup. The inner diameter of the adsorption reactor was $10 \mathrm{~mm}$. The toluene gas was generated by bubbling $\mathrm{N}_{2}$ into liquid toluene. $\mathrm{N}_{2}$ and air were the balance gases to maintain the desired toluene concentration and oxygen content. The oxygen content and the volumetric flow rate of feed were $10 \%$ and $500 \mathrm{sccm}$, respectively. After the inlet concentrations of toluene and oxygen reached a steady state, the adsorption of toluene was proceeded in the adsorption reactor. The detailed adsorption conditions are shown in Table I. An online GC (Shimadzu GC-2014) was used to monitor he toluene concentration at the inlet and outlet of the reactor. After equilibrium adsorption of toluene was achieved, the saturated adsorption capacity of toluene was calculated using numerical integration of the breakthrough curves, which represent the outlet concentrations of toluene versus time [16].

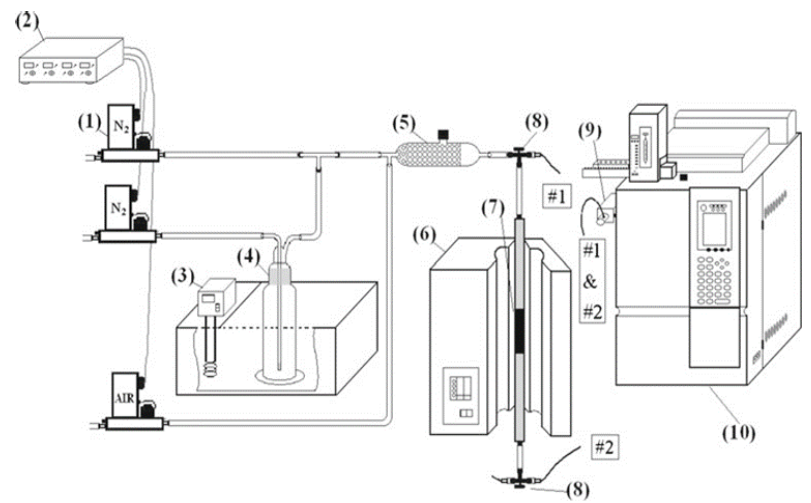

(1) Mass flow controller, MFC, (2) Power supply of MFC readout, (3) Thermostat water bath, (4) Gas bubbler filled with toluene, (5) Gas mixing chamber, (6) Heating unit, (7) Adsorption bed, (8) Three-way valve, (9) Gas sampling, (10) GC-FID

Fig. 1. Experimental setup for the adsorption of toluene.

\section{RESULTS AND DISCUSSIONS}

\section{A. Physical and Chemical Properties of Mesoporous Silica Mmaterials}

Fig. 2 shows the SEM image of mesoporous materials. SEM micrograph revealed that the particles of the synthesized mesoporous materials were crystalline and had a smooth surface. Fig. 3 shows the TEM image of mesoporous materials. TEM image showed that the hexagonal pore structure of mesoporous materials were unobvious. The result can be explained by the fact that other metal impurities are also present in the supernatant solution extracted from the bottom ash.

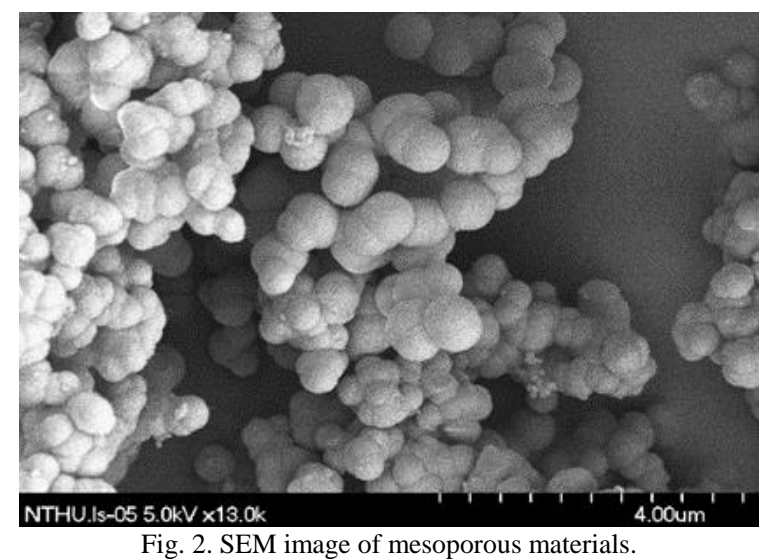

The BET results indicated that the specific surface area and mesoporous volumes of mesoporous materials were 992 $\mathrm{m}^{2} / \mathrm{g}$ and $0.854 \mathrm{~cm}^{3} / \mathrm{g}$, respectively. Fig. 4 and Fig. 5 display the $\mathrm{N}_{2}$ adsorption-desorption isotherms and the pore size distribution of the mesoporous materials. Fig. 4 indicated that the isotherm was of Type VI with an H1 hysteresis hoop due 
to the capillary condensation in mesopores [17], [18]. Further, the silica materials exhibited a narrow pore size distribution (as shown in Fig. 5). The average pore size of the mesoporous silica was $3.4 \mathrm{~nm}$.

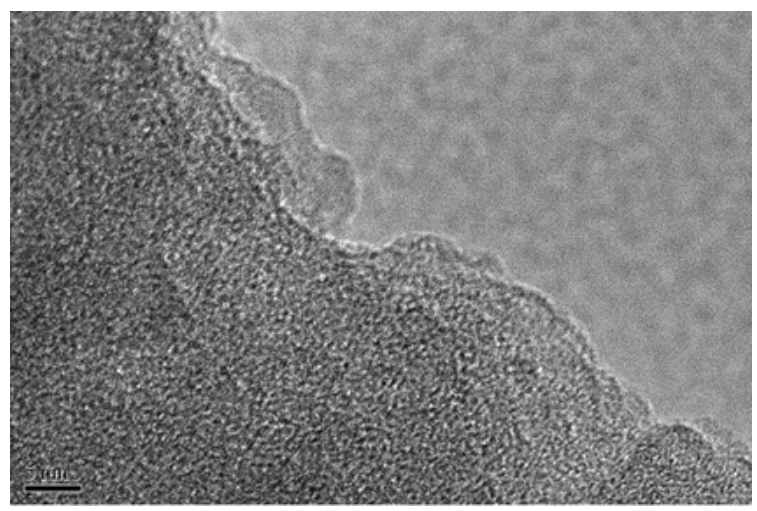

Fig. 3. TEM image of mesoporous materials.

Fig. 6 shows the XRD pattern of mesoporous materials. One peak corresponded to the (100) plane was observed in XRD patterns, indicating a hexagonal pore structure [19], [20]. Fig. 7 shows the TGA pattern of mesoporous materials. TGA patterns demonstrated that the synthesized mesoporous materials exhibited good thermal stability and hydrophobic property.

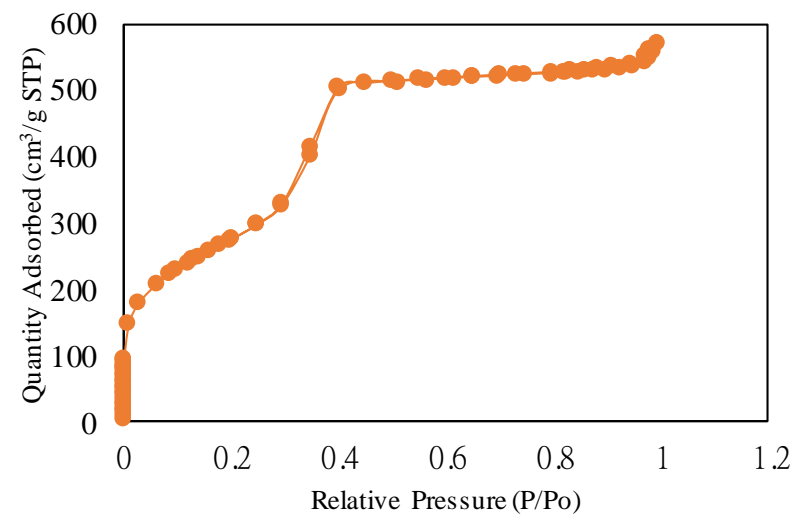

Fig. 4. $\mathrm{N}_{2}$ adsorption and desorption isotherms of mesoporous materials.

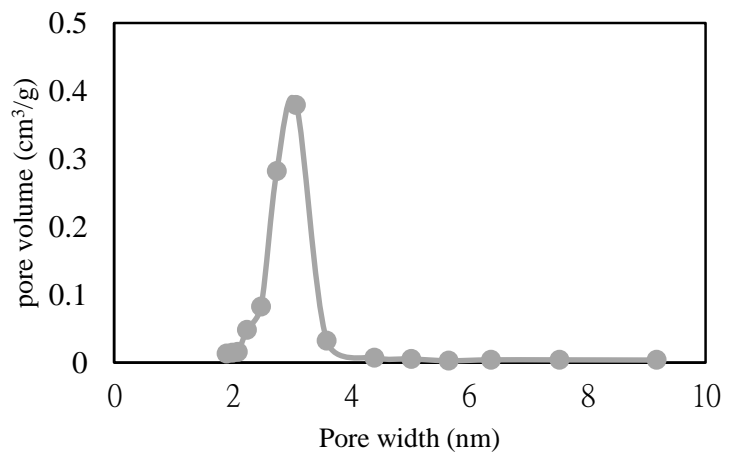

Fig. 5. Pore size distribution of mesoporous materials.

\section{B. Removal of Toluene}

Table I shows the effects of various adsorption conditions on the adsorption capacity of toluene. The mesoporous materials synthesized from bottom ash possessed the best adsorption capacity of toluene (ca. $124 \mathrm{mg} \mathrm{g}^{-1}$ ) at the toluene concentration of $1100 \mathrm{ppm}$ and at the reaction temperature of $25^{\circ} \mathrm{C}$. The data indicated that the adsorption capacity of toluene increased with increasing toluene concentration. The results also showed that the breakthrough times increased with increasing adsorbent weight, but the change in the adsorption capacity of toluene was insignificant. Ma and Ruan indicated that the adsorption capacity of toluene in mesoporous silica materials (S-MCM) synthesized from waste solar panel was 57, 104, 200 and $277 \mathrm{mg} \mathrm{g}^{-1}$ for initial toluene concentrations of 250 to $1500 \mathrm{ppm}$ respectively [21].

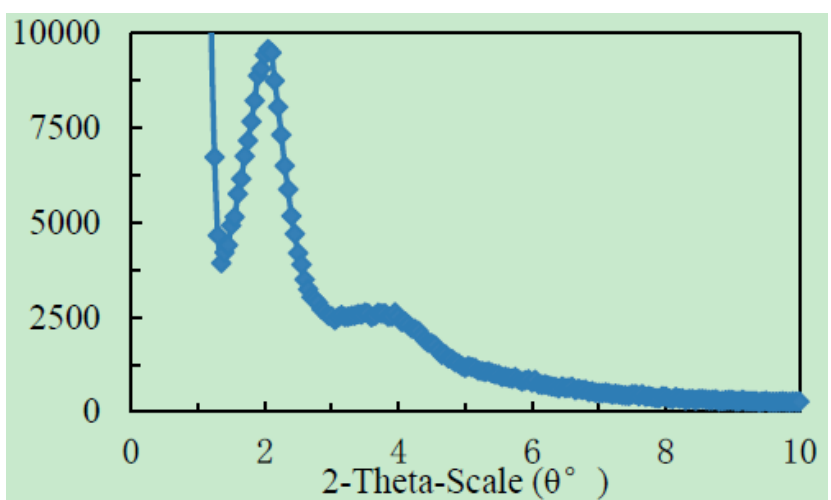

Fig. 6. XRD pattern of mesoporous materials.

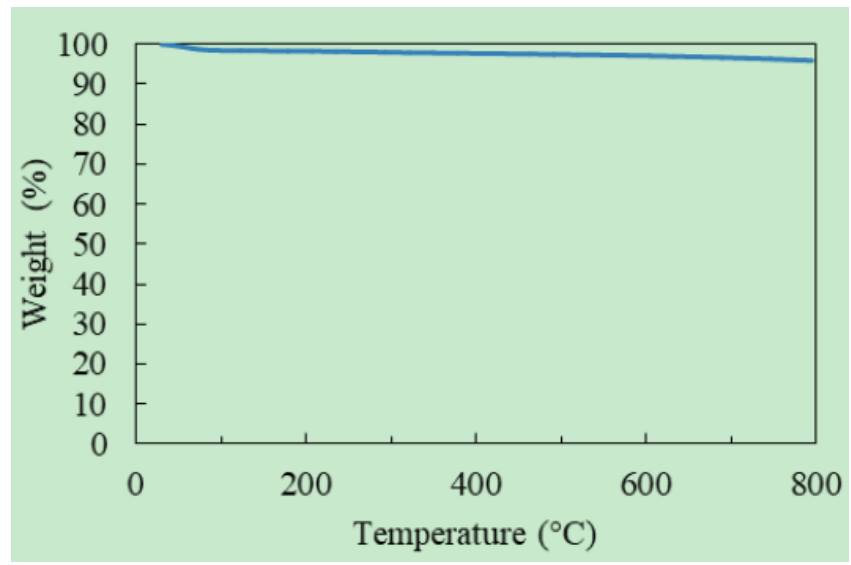

Fig. 7. TGA pattern of mesoporous materials.

\section{CONCLUSIONS}

This study investigated the synthesis of mesoporous silica materials from incineration ash for the removal of toluene in a fluidized-bed reactor. The results showed that the mesoporous silica has an optimum adsorption capacity of toluene (ca. $124 \mathrm{mg} \mathrm{g}^{-1}$ ) at the toluene concentration of 1100 ppm and at the reaction temperature of $25^{\circ} \mathrm{C}$. The mesoporous silica prepared with incinerator bottom ash has potential to be used as an adsorbent for removing toluene after being modified.

\section{ACKNOWLEDGEMENTS}

The author would like to thank the National Science Council of the Republic of China (Taiwan) for financially supporting this research under Contract No. MOST 104-2221-E-131-002-MY3.

\section{REFERENCES}


[1] P. Uma, and U. Fuangfa, "Simultaneous adsorption of silver nanoparticles and silver ions on large pore mesoporous silica," Journal of Environmental Chemical Engineering, vol. 6, pp. 596-603, 2018.

[2] E. S. Sanz-Péreza, T. C. M. Dantas, A. Arencibia, G. Calleja, A. P. M. A. Guedes, A. S. Araujo, and R. Sanz, "Reuse and recycling of amine-functionalized silica materials for $\mathrm{CO}_{2}$ adsorption," Chemical Engineering Journal, vol. 308, pp. 1021-1033, 2017.

[3] W. Zhu, J. Wang, D. Wu, X. Li, Y. Luo, C. Han, W. Ma, and S. He, "Investigating the Heavy Metal Adsorption of Mesoporous Silica Materials Prepared by Microwave Synthesis," Nanoscale Research Letters, vol. 12, pp. 323, 2017.

[4] L. Dolatyari, M. R. Yaftian, and S. Rostamnia, "Removal of uranium(VI) ions from aqueous solutions using Schiff base functionalized SBA-15 mesoporous silica materials," Journal of Environmental Management, vol. 169, pp. 8-17, 2016.

[5] H. Höller, and U. Wirsching, "Zeolites formation from fly ash," Fortschritte der Mineralogie, vol. 63, pp. 21-43, 1985.

[6] B. Wang, Y. Zhou, L. Li, and Y. Wang, "Preparation of amidoxime-functionalized mesoporous silica nanospheres (ami-MSN) from coal fly ash for the removal of U(VI)," Science of The Total Environment, vol. 626, pp. 219-227, 2018.

[7] B. Wang, Y. Zhou, L. Li, H. Xu, Y. Sun, and Y. Wang, "Novel synthesis of cyano-functionalized mesoporous silica nanospheres (MSN) from coal fly ash for removal of toxic metals from wastewater," Journal of Hazardous Materials, vol. 345, pp. 76-86, 2018.

[8] Y. R. Lee, J. T. Soe, S. Zhang, J. W. Ahn, M. B. Park, and W. S. Ahn, "Synthesis of nanoporous materials via recycling coal fly ash and other solid wastes: A mini review," Chemical Engineering Journal, vol. 317, pp. 821-843, 2017.

[9] G. Chandraseka, K. S. You, J. W. Ahn, and W. S. Ahn, "Synthesis of hexagonal and cubic mesoporous silica using power plant bottom ash," Microporous Mesoporous Material, vol. 111, pp. 455-462, 2008.

[10] Y. P. Ye, X. Q. Zeng, W. L. Qian, and M. W. Wang, "Synthesis of pure zeolites from supersaturated silicon and aluminum alkali extracts from fused coal fly ash," Fuel, vol. 87, pp. 1880-1886, 2008.

[11] M. Halina, S. Ramesh, M. A. Yarmo, and R. A. Kamarudin, "Non-hydrothermal synthesis of mesoporous materials using sodium silicate from coal fly ash," Materials Chemistry and Physics, vol. 101, pp. 344-351, 2007.

[12] M. Halina, S. Ramesh, B. Shahida, and A. Y. Mohd, "Processing of mesoporous silica materials (MCM-41) from coal fly ash," Journal of Materials Processing Technology, vol. 186, pp. 8-13, 2007.

[13] M. M. Liu, , L. A. Hou, B. D. Xi, Y. Zhao, and X.F. Xia, "Synthesis, characterization, and mercury adsorption properties of hybrid mesoporous aluminosilicate sieve prepared with fly ash," Applied Surface Science, vol. 273, pp. 706-716, 2013.
[14] Z. S. Liu, W. K. Li, and C. Y. Huang, "Synthesis of mesoporous silica materials from municipal solid waste incinerator bottom ash," Waste Management, vol. 34, pp. 893-900, 2014.

[15] R. V. Silva, J. Brito, C. J. Lynn, and R. K. Dhir, "Use of municipal solid waste incineration bottom ashes in alkali-activated materials, ceramics and granular applications: A review," Waste Management, vol. 68, pp. 207-220, 2017

[16] C. L. Lin, Y. H. Cheng, Z. S. Liu, and J. Y. Chen, "Adsorption and oxidation of high concentration toluene with activated carbon fibers," Journal of Porous Materials, vol. 20, pp. 883-889, 2013.

[17] S. J. Gregg, and K. S. W. Sing, "Adsorption Surface Area and Porosity," Academic Press, London, 1982.

[18] Z. Ryu, J. Zheng, M. Wang, and B. Zhang, "Characterization of pore size distributions on carbonaceous adsorbents by DFT," Carbon, vol. 37, pp. 1257-1264, 1999.

[19] H. Misran, R. Singh, S. Begum, and M. A. Yarmo, "Processing of mesoporous silica materials (MCM-41) from coal fly ash," Journal of Materials Processing Technology, vol. 186, pp. 8-13, 2007.

[20] Y. W. Chen and W. J. Wang, "Synthesis and characteristics of hexa-mesoporous silica: A review," Journal of Petroleum, vol. 39, pp. $17-36,2003$.

[21] C. M. Ma and R. T. Ruan, "Adsorption of toluene on mesoporous materials from waste solar panel as silica source," Applied Clay Science, vol. 80-81, pp. 196-20, 2013.

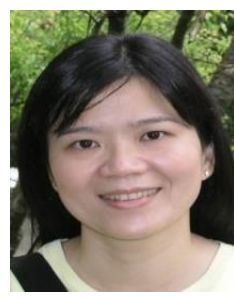

Zhen-Shu Liu is a professor in the Department of Safety, Health and Environmental Engineering at the Ming Chi University of Technology. Her expertise includes air pollution control, material synthesis, resource recovery, and environmental toxicology. Recently, her research focused on the assessment of cellular mutagenicity of foods and environmental pollutants. Her research findings addressed the above issues have been published in reputable journals.

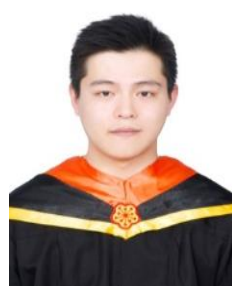

Hai-Ming Lan is a postgraduate student in the Department of Safety, Health and Environmental Engineering at the Ming Chi University of Technology. His Master's thesis focuses on the Synthesis of mesoporous silica materials from incineration bottom ash for the removal of indoor air pollutants such as toluene and formaldehyde. He is also interested in the studies about recovery of heavy metal from wastewater. 